\title{
Wirtschaftliche Beziehungen der Schweiz zu Lateinamerika : Aktuelles und Grundsätzliches
}

Les relations économiques de la Suisse avec l'Amérique latine: situation actuelle et tendances à long terme

Jean-Max Baumer

\section{OpenEdition}

\section{Journals}

Electronic version

URL: http://journals.openedition.org/sjep/998

DOI: $10.4000 /$ sjep.998

ISSN: 1663-9677

\section{Publisher}

Institut de hautes études internationales et du développement

Printed version

Date of publication: 1 janvier 1984

Number of pages: 195-209

ISSN: 1660-5926

\section{Electronic reference}

Jean-Max Baumer, «Wirtschaftliche Beziehungen der Schweiz zu Lateinamerika : Aktuelles und Grundsätzliches », Schweizerisches Jahrbuch für Entwicklungspolitik [Online], 4| 1984, Online erschienen am: 28 Mai 2013, abgerufen am 08 September 2020. URL : http://journals.openedition.org/sjep/998 ; DOI : https://doi.org/10.4000/sjep.998 


\title{
WIRTSCHAFTLICHE BEZIEHUNGEN DER SCHWEIZ ZU LATEINAMERIKA : AKTUELLES UND GRUNDSAETZLICHES
}

\author{
Jean-Max Baumer
}

Ich danke Herrn Peter Kahane, Assistent am ILE, für die produktive Zusammenarbeit, seine zahlreichen Vorarbeiten und für seine Niederschrift eines Entwurfs, der mir in Aufbau und Inhalt eine unverzichtbare Grundlage bot.

\section{RESUME : Les relations économiques de la Suisse avec l'Amérique latine : situation actuelle et tendances à long terme}

Dans cet article, l'auteur examine en particulier trois éléments des liens économiques que la Suisse entretient avec I'Amérique latine : les relations commerciales, les investissements directs suisses et la participation de la Suisse à la coopération financière internationale pratiquée en faveur des pays latino-américains. II arrive aux conclusions suivantes :

Les échanges commerciaux de la Suisse avec l'Amérique latine représentent une part très modeste du commerce extérieur suisse et ils tendent à baisser à long terme.

Les échanges commerciaux avec l'Amérique latine, traditionnellement caractérisés par l'achat de matières premières et la vente de produits manufacturés suisses, présentent régulièrement un excédent en faveur de la Suisse, comme c'est le cas plus généralement pour les échanges commerciaux de la Suisse avec l'ensemble du Tiers Monde. Trois pays, le Mexique, l'Argentine et le Brésil, reçoivent à eux seuls $50 \%$ des exportations suisses à destination de l'Amérique latine et absorbent $60 \%$ des engagements au titre de la GRE accordée par la Confédération pour les exportations vers ce continent. Pour des raisons diverses, la capacité d'importation de ces pays a fortement diminué ces dernières années, et ils accumulent des dettes extérierues très importantes.

Près de $60 \%$ des investissements privés suisses sont placés en Amérique latine (leur montant étant estimé à environ 5 milliards de dollars). La Suisse est, derrière les Etats-Unis et la RFA, le troisième investisseur dans les pays latino-américains. Les investissements directs présentent ainsi un intérêt primordial pour la Suisse sur le continent latino-américain; ils tendent à remplacer en partie les produits antérieurement exportés par la Suisse. Sur le total de 5 milliards de dollars, 3 milliards sont placés au Mexique, en Argentine et au Brésil. Depuis les années 1981-83, les flux de capitaux suisses vers I'Amérique latine ont toutefois considérablement diminué (endettement croissant, instabilité économique, etc.). Malgré les problèmes existants, l'Amérique latine restera, à long terme, un marché très attractif pour les investisseurs suisses. II s'agira de maintenir la position suisse sur les marchés futurs face à la concurrence internationale.

Dans le cadre de la coopération financière internationale, les banques suisses participent activement aux efforts déployés en faveur de l'Amérique latine. La Suisse est (depuis 1976) membre de la Banque interaméricaine de développement et représente pour la BID 
le deuxième marché de capitaux après les Etats-Unis. La Suisse participe en outre aux crédits transitoires accordés par la Banque des règlements internationaux en faveur des pays latino-américains, aux Accords généraux d'emprunt ( $A G E$ ) élargis du FMI et aux négociations menées au sein du Club de Paris en vue de la consolidation des dettes des pays en développement.

En tant que petit pays par rapport à d'autres pays industrialisés, la Suisse assure une présence économique très importante en Amérique latine. Elle restera un partenaire solide, intéressé à une évolution favorable à long terme de ses relations avec les pays latinoaméricains.

\section{HANDELSBEZIEHUNGEN}

Lateinamerika ist ein mit Rohstoffen und Landfläche gesegneter Kontinent; die Schweiz stellt in beiden Beziehungen das Gegenteil dar, und ist - pro Kopf gerechnet - weltweit der grösste Importeur landwirtschaftlicher Erzeugnisse.

Die Handelsbeziehungen mit Lateinamerika reichen daher weit ins letzte Jahrhundert zurück. Frappant ist jedoch der Umstand, dass unser Aussenhandel mit Lateinamerika im Verhältnis zum gesamten schweizerischen Aussenhandel sehr bescheiden und überdies langfristig im Sinken begriffen ist. Die ursprüngliche Form der Arbeitsteilung - Einkauf von Rohstoffen und Absatz von verarbeiteten Erzeugnissen - blieb mehr oder weniger bestehen. Das Tonnenpreisverhältnis der Exporte zu den Importen im schweizerischen Aussenhandel allgemein beträgt $6: 1$; im Handelsverkehr mit Mexiko, Brasilien und Argentinien erreicht das Verhältnis gar 12:1 (OZD, 1982). ।

Die Schweiz weist gegenüber Lateinamerika regelmässig einen Handelsbilanzüberschuss auf, analog zum traditionellen Ueberschuss gegenüber der ganzen Dritten Welt. Im Ausmass dieses "Importmangels" kreditieren wir regelmässig unsere Handelspartner, ein Aspekt, der lange Zeit nicht viel Beachtung fand. 1981 wies die Schweiz gegenüber Lateinamerika einen Rekord-Ueberschuss von 1,5 Mia Franken auf; 1983 betrug dieser noch 720 Mio Franken.

\section{Exporte}

Die Warenexporte nach Lateinamerika bezifferten sich 1983 auf 1,8 Mia Franken (Léchot, 1984) oder $14 \%$ unserer Exporte in die Dritte Welt bzw. $3,3 \%$ aller schweizerischen Exporte. 1982 hatten die Exporte nach Lateinamerika noch etwas mehr als 2,2 Mia Franken ausgemacht. 
Vom Exportrückgang besondern betroffen wurden letztes Jahr die traditionellen Exportbereiche Maschinenbau, Instrumente und Apparate (- 100 Mio Fr.), Uhren (- 65 Mio Fr.) und Textilien (- 8 Mio Fr.). Chemikalien und Pharmaprodukte, hinter Maschinen- und Instrumentenbau die zweitwichtigste Exportposition, vermochten die Vorjahresposition auch $1983 \mathrm{zu}$ halten.

$50 \%$ unserer Exporte gehen nach Mexiko, Argentinien und Brasilien, also die Länder, deren Importkapazität in allerletzter Zeit infolge ihrer ungenügenden internationen Liquidität besonders drastisch sank.

\begin{tabular}{lccc}
\hline Länder & \multicolumn{1}{c}{$\begin{array}{c}\text { Exporte der Schweiz in Mio Fr. } \\
\text { 1982 }\end{array}$} & $\mathbf{1 9 8 3}$ & Rückgang \\
& & & \\
Argentinien & 355 & 228 & $-36 \%$ \\
Mexiko & 410 & 181 & $-56 \%$ \\
Brasilien & 414 & 383 & $-8 \%$ \\
\hline
\end{tabular}

Ohne den absoluten Umfang unserer Exporte nach Lateinamerika geringschätzen zu wollen, muss doch dessen geringe relative Bedeutung für die Schweiz betont werden.

\begin{tabular}{lcc}
\hline Jahre & $\begin{array}{c}\text { Exporte der Schweiz nach } \\
\text { Lateinamerika in Mio Fr. }\end{array}$ & $\begin{array}{c}\text { Anteil am Gesamtexport } \\
\text { der Schweiz in \% }\end{array}$ \\
1960 & 630 & 7,8 \\
1965 & 755 & 5,9 \\
1970 & 1335 & 6,0 \\
1975 & 1910 & 5,7 \\
1980 & 2290 & 4,7 \\
1981 & 2690 & 5,1 \\
1982 & 2230 & 4,4 \\
1983 & 1800 & 3,3 \\
(OZD, diverse Jg.) & \\
\hline
\end{tabular}




\section{Relativ sinkender Aussenhandel}

Die zwei wichtigsten Gründe für diese Entwiclung liegen bei der Importsubstitutionspolitik einerseits und der zyklischen Devisenverfügbarkeit der Länder Lateinamerikas anderseits.

Praktisch alle südamerikanischen Länder haben vor rund einem halben Jahrhundert ihre wirtschaftspolitische Betonung auf die Binnenwirtschaftspolitik gelegt und - nicht ohne Erfolg - versucht, die Konsumprodukte sowie nach und nach auch die Vorprodukte, Zwischenprodukte und Investitionsgüter selbst herzustellen. Die hierzu erforderlichen Beiträge von aussen wechselten entsprechend ihren Charakter : von "Gütern" zu immer mehr "Dienstleistungen" (vor allem technische Assistenz und Lizenzen) und "Kapital" (Direktinvestitionen und andere). Die schweizerischen Direktinvestitionen, fast ausschliesslich auf die dortigen Binnenmärkte gerichtet, ersetzen ihrerseits frühere klassische Exporte aus der Schweiz. In einzelnen Bereichen (z.B. Pharma) gelang allerdings die Aufrechterhaltung von Exporten aus der Schweiz nur mit Hilfe von Direktinvestitionen; die Ausfuhr von chemischen Grundsubstanzen bzw. Wirkstoffen wären sonst auch gefährdet. Die aussenhandelsmässige Bedeutungslosigkeit Lateinamerikas für die Schweiz hat daher verschiedene Gesichter; sie ist Ausdruck der stetig wachsenden Selbstversorgungsfähigkeit des Kontinents im Bereich der Konsum- und vieler Investitionsgüter, aber sie zeigt auch die wachsende Bedeutung der "vorgelagerten Märkte", also alle "soft- und hardware"-Transfers für die Herstellung von Investitions- und Kollektivgütern aller Art.

Die unregelmässige Devisenverfügbarkeit ihrerseits entsteht durch die immer noch stark auf die Rohstoffe ausgerichtete Exportwirtschaft Lateinamerikas (ungefähr $90 \%$ aller Exporte) mit den bekannten Preisabhängigkeiten und Importbegrenzungen der Industrieländer für land- und viehwirtschaftliche, aber auch für industrielle Güter. Kombiniert man beide Ursachen der Aussenhandelsverminderung, so ist unschwer zu verstehen, dass lateinamerikanische Länder Devisenmangel praktisch nur mit Importbegrenzungen wettmachen können. In der Regel geraten jene Importkategorien unter die Räder, die der "similar nacional"-Vorstellung am stärksten entsprechen. Gegenwärtig haben die grossen, besonders hochverschuldeten Länder ihre Importe über das Mass eigener Substitutionsmöglichkeiten hinaus begrenzen müssen, womit sie (besonders Mexiko) ihr internes industrielles Wachstum für die nächsten Jahre behindern. Es wird an spezifischen Rohstoffen, Halbfabrikaten und Spezialeinrichtungen fehlen. Dem aussenwirtschaftlichen Engpass folgt ein binnenwirtschaftlicher, welcher wiederum einer Belebung des Aussenhandels hinderlich sein wird. In der Regel korreliert der Liberalisierungsgrad lateinamerikanischer Importpolitik gut mit der Devisenverfügbarkeit, ihrerseits Resultat der Exporterlöse sowie des Nettozuflusses von Auslandskapital.

Zu diesen zwei grundlegenden Ursachen der Aussenhandelskomprimierung gesellen sich weitere negative Faktoren wie die allgemeine Investitionsstille, 
die ungewöhnlichen Austeritätsprogramme der Regierungen (mit Staatsanteilen am BIP bis zu $70 \%$ ) und die Ausbreitung bilateraler Kompensationsgeschäfte (NZZ, 16.-17.6.84) südamerikanischer Staaten, was besonders Handelspartner mit Exportüberschüssen wie die Schweiz zur Vermehrung der Importe oder Senkung der Exporte "zwingt". Oekonomisch gesehen wird es auch keine andere Lösung geben : handeln heisst tauschen, und auf die Länge gesehen müssen alle Länder zum besseren Ausgleich der Güter- und Dienstleistungsbilanzen Lateinamerikas beitragen. Lateinamerika muss seine Schuldendienstzahlungen und eigenen Importe auf die Länge erwirtschaften können.

Das setzt auch voraus, dass Lateinamerika der Schweiz konkurrenzfähige Importangebote macht, ein Problem auf das wir zurückkommen. Der schweizerische Importmangel aus Lateinamerika behindert jedenfalls schon heute die eigenen Exportmöglichkeiten.

\section{ERG}

Dieser Tatbestand lässt sich auch mit der Exportrisikogarantie - ERG - nicht aus der Welt schaffen. Zwar ist die ERG ein hilfreiches Instrument zur kurzfristigen Exportförderung, aber sie erhöht nur das Exportangebot, nicht die schweizerische Importnachfrage. Es ist daher auch nicht erstaunlich, dass das Gesamtengagement der ERG sukzessive wächst : 1984 (Frühjahr) : $18 \mathrm{Mia}$ Franken, davon 3 Mia Franken für Lateinamerika im Vergleich zu 1964 : 1,4 Mia Franken, davon 420 Mio Franken für Lateinamerika (Léchot, 1984). Die Exporte der Schweiz nach Lateinamerika wuchsen seit 1964 um rund das Zweieinhalbfache, die ERG-Zusagen um mehr als das Siebenfache. Der Bund leistete 1982 einen Vorschuss an die (ganze) ERG von 109 Mio Franken, 1983 bereits 240 Mio Franken und 1984 dürften die Zuschüsse noch grösser ausfallen (Bundesrat 1984, p. 28).

Man kann das ERG-Engagement mit Blick auf die Beschäftigungswirkungen im eigenen Exportsektor weiter ausdehnen, doch sollte man nicht vergessen, dass letzlich die Steuerzahler die Rechnungen begleichen. Volkswirtschaftlich gesehen könnte man mit diesem Geld auch lateinamerikanische Güter kaufen und damit deren Importkapazität stärken; diese käme teilweise den schweizerischen Exporteuren ohne Zweifel wieder zugute.

ERG-unterstützte Exporte nach Lateinamerika sind übrigens aus versicherungstechnischen Gründen Grenzen gesetzt durch die länderweisen ERGPlafonds, besonders aber durch den Umstand, dass bereits $60 \%$ der Engagements auf Argentinien, Brasilien und Mexiko entfallen (Léchot, 1983). Sie sind die grössten Abnehmer, weisen aber die höchsten Schulden auf und haben den ERG-Spielraum bereits stark ausgeschöpft. 


\section{Importe}

Unsere traditionellen Einfuhrprodukte aus Lateinamerika sind Kaffee (zwei Drittel unseres Bedarfs), Fleisch (ein Drittel unseres Bedarfs), Bananen (ganzer Bedarf der Schweiz), Tabak, Rohbaumwolle, Kakao und Edelsteine.

Seit 1960 haben sich die Importe rund verdreifacht, aber sie betragen im Durchschnitt der letzten zwanzig Jahre nur 50 \% der schweizerischen Exporte nach Lateinamerika. Genau wie die Exporte gingen die Importe seit 1981 leicht zurück.

\begin{tabular}{lcc}
\hline Jahre & $\begin{array}{c}\text { Importe der Schweiz aus } \\
\text { Lateinamerika in Mio Fr. }\end{array}$ & $\begin{array}{c}\text { Anteil am Gesamtimport } \\
\text { der Schweiz in \% }\end{array}$ \\
1960 & 375 & 3,8 \\
1965 & 470 & 3,0 \\
1970 & 640 & 2,3 \\
1975 & 745 & 2,2 \\
1980 & 1195 & 2,1 \\
1981 & 1190 & 2,0 \\
1982 & 1040 & 1,8 \\
1983 & 1080 & 1,8 \\
\hline
\end{tabular}

\section{Schlechte Wettbewerbsfähigkeit}

Lateinamerika hat langfristig weltweit Exportmarktverluste erlitten. 1948 betrug der Weltmarktanteil $12 \%, 1980$ noch $4,5 \%$ (SALA, vol. 22). Hauptverantwortlich für diese Entwicklung ist der durch hohe Zölle und andere Massnahmen (überbewertete Währungen, Importkontingente, etc.) erreichte "Ueberschutz" der eigenen Industrien. Die Produktionskosten sind zu hoch, die Angebotsstruktur ist wenig diversifiziert, die Lieferkonstanz ist mangelhaft. Vor allem ist das Wachstumspotential der klassischen lateinamerikanischen Ausfuhren (Rohstoffe) sehr beschränkt (Petersen, 1983). Die"vier grossen Asiaten" Hongkong, Singapur, Taiwan und Südkorea eroberten dank höherer Arbeitsproduktivität, exportfreundlicherer Wechselkurse, Produktqualität, aggressiver Markterschliessung und der Fähigkeit zur kurzfristigen Anpassung an Nachfrageänderungen neue Weltmärkte.

Der schweizerische Zolltarif würde industrielle Importe aus Lateinamerika wegen der gewährten Präferenzzölle begünstigen, währent nur ein Sechstel agrarischer Erzeugnisse präferentielle Behandlung erfährt. 1980 erreichte aber der Ausnutzungsgrad der gewährten Industrieprodukte-Präferenzen nur 
$25 \%$, da offensichtlich das entsprechende Angebot fehlte. Der Vergleichswert für Agrarprodukte betrug $85 \%$ (Handelskammer, 1980).

Schliesslich ist daran zu erinnern, dass schweizerische Exporte und Importe privatwirtschaftliche Tätigkeiten sind. So wünschenswert und nötig eine Erhöhung der Importe aus Lateinamerika auch ist, lässt sie sich doch nicht durch staatlichen Zwang oder öffentliche Kompensationsgeschäfte bewerkstelligen.

\section{Zusammenfassung}

- Der Aussenhandel mit Lateinamerika betrug 1983 als Anteil am gesamten Aussenhandel der Schweiz noch die Hälfte gegenüber 1960; gleichzeitig verdoppelte sich der Aussenhandelsanteil mit den "vier grossen Asiaten".

\begin{tabular}{lcccc}
\hline & \multicolumn{2}{c}{ Exportanteile } & \multicolumn{2}{c}{ Importanteile } \\
& LA & “Asien" & LA & “Asien" \\
1960 & $7,8 \%$ & $10,0 \%$ & $3,8 \%$ & $3,7 \%$ \\
1983 & $3,3 \%$ & $16,8 \%$ & $1,8 \%$ & $8,0 \%$ \\
(OZD) & & & & \\
\hline
\end{tabular}

- Die schweizerischen Exporte nach Lateinamerika liegen $20 \%$ tiefer als unsere Ausfuhren nach Oesterreich; die Importe aus Lateinamerika erreichen nur die Hälfte unserer Einfuhren aus Oesterreich.

- Die Möglichkeiten der ERG zur Exportbelebung nach Lateinamerika sind weitgehend aufgebraucht.

- Die lateinamerikanischen Exportangebote sind für die schweizerischen Importeure nicht allzu attraktiv; eine Erhöhung der schweizerischen Importe zur Verbesserung der Leistungsbilanz stösst auf Schwierigkeiten.

- Die Handelsaussichten sind infolge externer Verschuldungen und interner Rezessionen nicht besonders erfreulich. 


\section{SCHWEIZERISCHE DIREKTINVESTITIONEN}

\section{Starke Präsenz}

Selbst mit konservativen Schätzungen über das Ausmass schweizerischer Direktinvestitionen (1978 : 3,4 Mia \$ gemäss NZZ 1983, 1982 : 4 Mia \$ gemäss Bundesamt für Aussenwirtschaft) in Lateinamerika, erreicht die Schweiz international den 3 . Platz mit 7,5\% aller privaten Auslandsinvestitionen in Lateinamerika. An erster Stelle sind die USA mit $48 \%$, gefolgt von der BRD mit 8,5\%; hinter der Schweiz folgen Japan, Kanada, Grossbritannien und Frankreich.

Die schweizerischen Privatinstitutionen in Lateinamerika - sie dürften um die 5 Mia $\$$ betragen - entsprechen rund $60 \%$ unserer Direktinvestitionen in der Dritten Welt. Hier also liegt ein Hauptinteresse unseres Landes am iberoamerikanischen Kontinent. Die Schwerpunkte unserer Investionen liegen in den drei Ländern mit grossen Binnenmärkten und guter industrieller Diversifikation (Esser/von Gleich, p. 48); 3 Mia \$ entfallen auf Mexiko, Argentinien und Brasilien, auf letzteres allein 2 Mia \$ (SALA, p. 509). $85 \%$ unserer Investitionen betreffen verarbeitende Industrien, vor allem Maschinenbau, Chemie und Pharma, Nahrungsmittel und Baustoffe.

Zwar haben schweizerische Direktinvestitionen seit 1920 stattgefunden, erfolgte eine spürbare Verstärkung zwischen 1955 und 1970 (Enderlin, p. 140) sowie eine weitere Beschleunigung ab 1970 bis 1981 (Esser/von Gleich, p. 48). Häufig erfolgten die Investitionen in drei Stufen : unabhängige Agentur oder Repräsentant, firmeneigene Verkaufsorganisation mit "Abfüllbetrieben" und schliesslich lokale Fabrikation. Seit 1981, besonders aber 1982-83 liess der Investitionsmut in Lateinamerika allgemein nach; das im letzten Jahr sehr zurückhaltende Bankensystem und die abwartenden, auf Sparflamme operierenden Unternehmen liessen die Kapitalzuflüsse stark zurückgehen. Gleichzeitig stiegen in den letzten Jahren die Zinsen auf bestehenden und neuen Schulden um $30 \%-50 \%$. Besondern das Wissen der Geschäftswelt, dass die Schuldenkonsolidierungen mit oder ohne Hilfe des IWF gar keine mittelfristige Lösung, sondern eine Kombination von Ratlosigkeit und Zukunftshoffnung darstellen, nährt die momentane Skepsis. Direktinvestoren haben auch in besseren Zeiten schon so erhebliche Transferprobleme bezüglich Technologiegebühren, Transferpreisen, Zinsen und Dividenden sowie ungenügenden Registrierungsmöglichkeiten von Reinvestitionen erlebt, dass sie sich fragen, was denn in schlechten Zeiten noch auf sie zukommen mag. Die drastischen Importsperren mit Strangulationswirkungen für einzelne industrielle Sparten in Lateinamerika wurden weiter oben bereits besprochen (vgl. Teil I, Exporte). 


\section{Schweizerische Investoren : Verschieben, aber nicht aufgeben}

Trotz dieser Probleme behält Lateinamerika in den Augen schweizerischer Investoren - langfristig - hohe Attraktivität. Die Erklärung liegt in erster Linie im ungebrochenen Glauben an die Zukunft dieser Märkte und in zweiter Linie im Einnehmen oder Festhalten einer relativ starken Marktposition. Wer heute aufgibt, ist morgen - bei besserer Situation - nicht aktionsfähig. Die starke Präsenz in Zukunftsmärkten sind die zwei wichtig sten Motive schweizerischer Direktinvestoren in Lateinamerika, während in den "vier grossen Asiaten" komparative Kostenvorteile für den Kampf auf Weltmärkten gesucht werden. Der Konkurrenzaspekt spielt bei schweizerischen Direktinvestitionen in Lateinamerika allerdings auch eine, wenngleich sehr spezifische Rolle : die Eroberung U.S.-amerikanischer Marktanteile. Nur rund ein Drittel der U.S.-Direktinvestitionen in Lateinamerika betreffen verarbeitende Industrien, aber Lateinamerika importiert solche Produkte überwiegend aus USA. Schweizerische und deutsche TNC's konkurrieren dank ihrer lokalen Produktion erfolgreich gegen die amerikanischen Exporte (European Challenge, 1982, p. 134). Brasilien ist für viele TNC's nach den USA das bedeutendste Investitionsziel in Uebersee (SHZ, 16.11.78).

\section{Ungünstige Rahmenbedingungen}

Die Binnenrezessionen in Lateinamerika haben die Ertragslage schweizerischer Firmen zwar stark beeinträchtigt, aber die wirklichen Probleme sehen die Investoren in den unübersehbar gewordenen gesetzlichen Restriktionen, der bürokratischen Verschleppung und Komplizierung (und Verteuerung) des Tagesgeschäftes und den politischen, besonders wirtschaftspolitischen Unstabilitäten. Die bereits erwähnten Transferschwierigkeiten, die JointVenture-Auflagen, der ungenügende Schutz von Know-how und von Handelsmarken, Exportzwänge, Preisblockierungen trotz galoppierender Inflation bis hin zu Verstaatlichungen sind Beispiele dieser Problemfelder.

Gegenwärtig ist eine gewisse Gegentendenz zu beobachten; so werden beispielsweise die einschneidenden Vorschriften im Andenpaktstatut bezüglich "Fade-out-Joint-Ventures" revidiert, um neue Anreize zu schaffen (NZZ, 22. 5.84). Erfahrene Investoren werden sich allerdings daran erinnern, dass Liberalisierungstendenzen regelmässig interventionistische Phasen folgten. Die Behandlung des Auslandskapitals richtet sich stärker nach der Lage der Zahlungsbilanz als nach einem durchdachten, stabilen Konzept. Die Frage, weshalb unseren und anderen Investoren die Lust am Aufbau von Produktivkapital nicht ausgeht, lässt sich m.E. so beantworten : die Motive der Investoren vereinigen sich mit den Absichten der Empfangsländer zu einer komplexen Konfiguration von Zielen. Das Ziel der TNC's in zukunftsträchtigen Märkten zu operieren, verträgt sich gut mit den Modernisierungsplänen der 
Empfangsländer; sie brauchen dafür die moderne Technologie und das Marketing Know-how der TNC's. Während diese aber - zweites Hauptmotiv eine starke Marktstellung anstreben, versuchen die Empfangsländer, die nationale Kontrolle über das Wirtschaftsgeschehen zu vermehren. In diesem Punkt kollidieren die beidseitigen Interessen. Die Resultante ist ein unklarer, gespaltener wirtschaftspolitischer Kurs; einmal haben die TNC's eher die Oberhand, ein anderes Mal die Regierungen. Die Lage der Zahlungsbilanz indessen ist ein recht verlässlicher Indikator. Allerdings haben auch die TNC's kein Interesse an strukturellen oder liquiditätsmässigen Krisen der Zahlungsbilanzen, selbst wenn diese kurzfristige gewisse Behandlungserleichterungen bringen.

\section{Bilaterale Schutzverträge}

Entscheidend wichtig sind einigermassen gleichbleibende Spielregeln, ein klarer rechtlicher Rahmen, politische Sicherheit und vor allem ein klares Anforderungsprofil an die Adresse der TNC's. Zur Verbesserung dieser Komponenten schliesst die Schweiz bilaterale Verträge über den Schutz und die Förderung von Kapitalinvestitionen ab (Botschaft, 1982). Die Schweiz bemüht sich im Rahmen dieser Abkommen mit Entwicklungsländern für Investoren vor allem Regelungen der Transfers und Garantien im Falle von Verstaatlichung zu sichern.

Seit 1961 wurden 34 solche Verträge abgeschlossen, 23 mit Ländern Afrikas, 7 mit asiatischen Ländern, 1 Vertrag mit Malta und nur 3 mit lateinamerikanischen Ländern (Costa Rica 1965, Honduras 1966, Ecuador 1968). Die für die Schweiz wirklich relevanten Länder verweigern den Abschluss solcher Verträge mit der Begründung, sie stellten Eingriffe in ihre nationale Souveränität dar (Botschaft, 1982).

\section{Zusammenfassung}

Die nachfolgende Tabelle zeigt die langfristige Entwicklung der prozentualen Anteile diverser privater Kapitalflüsse (ganzes Ausland) nach Lateinamerika (eine spezifisch schweizerische Zusammenstellung ist nicht erhältlich). Die Tendenzen stimmen aber auch für die schweizerischen Verhältnisse.

Die Geschäftsbanken rutschten offensichtlich in den letzten zehn Jahren in die Rolle der Entwicklungsbanken. Mit diesem Problemkreis befasst sich der nächste Abschnitt. 


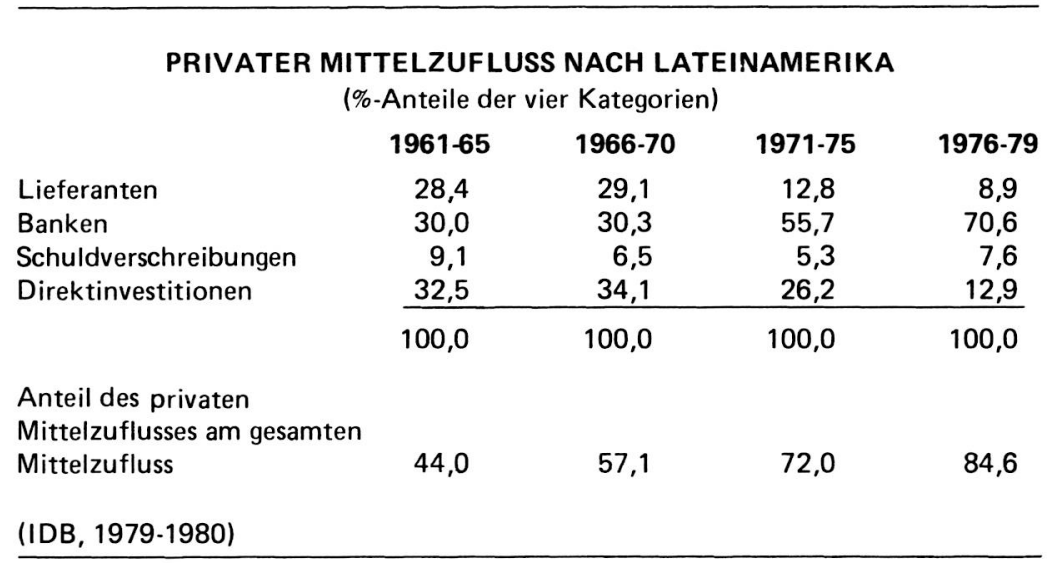

\section{BANKENFINANZIERUNG UND VERSCHULDUNG}

\section{Stellung der Schweizer Banken}

Die Grossbanken, die in der Schweiz domizilierten Auslandsbanken sowie die ausländisch beherrschten Finanzgesellschaften verfügten 1982 über 23 Mia Franken in 60 Problemländern, davon 13 Mia Franken in Lateinamerika; hiervon stammen 10 Mia Franken von schweizerischen Grossbanken (Bundesrat, 1984). Dieses Engagement entspricht lediglich $2 \%$ der Bilanzsumme dieser vier Institutionen (HEBDO, 23.12.82), und ist im Rahmen der stillen Reserven und Rückstellungen bewältigbar. Bis Ende 1984 sollen die Vorkehrungen getroffen sein, um diese Ausstände abzuschreiben (Wall Street Journal, 10.4.84).

Ohne Zweifel verfügt das schweizerische Bankensystem über eine weltweit gute Streuung ihrer Auslandsguthaben.

Trotz Diversifikation der Guthaben und reichlichen Reserven wird natürlich bei steigendem Rückstellungsbedarf das potentielle Steueraufkommen vermindert. Aber immerhin : das Verhältnis der betreffenden Engagements zu den eigenen Mitteln beträgt für die Schweizer Banken 0,5, für die amerikanischen Banken mehr als 1.

Eine andere Frage betrifft die Risiken der schweizerischen Banken im Interbank-Geschäft. Eine Spezialität unserer Banken besteht darin, grosse inter- 


\section{GLIEDERUNG DER AUSLANDSGUTHABEN NACH LAENDERGRUPPEN PER ENDE 1982}

\begin{tabular}{lcrrr} 
& \multicolumn{2}{c}{$\begin{array}{c}\text { Alle Banken und Finanz- } \\
\text { gesellschaften }\end{array}$} & \multicolumn{2}{c}{ Grossbanken } \\
& in Mia Fr. & in $\%$ & in Mia Fr. & in \% \\
BIZ-Länder & 152,0 & 67,4 & 102,2 & 69,9 \\
übriges Westeuropa & 16,1 & 7,1 & 8,2 & 5,6 \\
Osteuropa & 4,7 & 2,1 & 2,1 & 1,4 \\
Karibik & 14,9 & 6,6 & 9,2 & 6,2 \\
Lateinamerika & 13,3 & 5,9 & 9,6 & 6,5 \\
Afrika & 7,8 & 3,5 & 4,3 & 2,9 \\
Mittlerer Osten & 6,3 & 2,8 & 3,7 & 2,5 \\
Asien & 10,3 & 4,6 & 8,6 & 5,8 \\
\cline { 2 - 5 } & 225,4 & 100,0 & 147,9 & 100,0
\end{tabular}

(Bundesrat, 1984)

nationale Geschäftsbanken mit kurzfristigem Kapital zu versorgen, die ihrerseits Länderrisiken eingehen. Daraus entsteht ein Risiko 2. Grades (Bundesrat, 1984). Hierin verbergen sich durch pyramidale Verflechtungen grössere Risiken als in den eigenen Länderrisiken.

Der schweizerische Notenbankenchef Leutwiler schlägt als Lösungsansatz für die lateinamerikanischen Schuldenprobleme die Aushandlung neuer langfristiger Bankkredite, verbunden mit Zinserleichterungen vor (TA, 16.4.84). Diese Ansicht scheint im schweizerischen Geschäftsbankensystem weit verbreitet, wenn auch nicht unbestritten zu sein (Stellungnahme, 3/84). Schwieriger haben es die Lateinamerikaner mit den amerikanischen Banken, deren Interessenlage andersartig ist (NZZ, 27.4.84). Hier steht der Zinsendienst im Vordergrund. Ihre gesetzlichen Bestimmungen betreffend die Debitorenbewertung verunmöglicht ihnen praktisch, Zinsfälligkeiten länger als 90 Tage anstehen zu lassen, und da ihre Rückstellungen dürftig sind, bangen sie bei jedem Quartalsultimo um ihre kurzfristigen Gewinnausweise. Diese schlagen schnell auf die Börse durch.

Die europäischen Banken verfügen über einen erheblich grösseren Bilanzierungsspielraum und mehr Reserven. Statt kurzfristige Sicherung des Zinsendienstes zu erzwingen, ziehen sie es vor, auf Schuldenkonsolidierungsprogramme einzutreten, und kein Karussell kurzfristigen Neugeldes für kurzfristig fällig werdende Zinsen in Gang zu setzen.

Die lateinamerikanischen Schulden haben 1983 zur Gründung eines privatwirtschaftlichen "Institute for International Finance" mit Sitz in New York 
geführt, welches von 35 international führenden Banken, auch zwei schweizerischen Grossbanken, getragen wird. Dadurch soll die Transparenz internationaler Kapitalmärkte erhöht und Gedankenarbeit für die Zukunft geleistet werden.

\section{Beziehungen zur "Interamerican Development Bank" (IDB)}

Die IDB wurde 1959 gegründet. Erst 1966 gestattete die Schweiz eine erste Kapitalmarktaufnahme, vor allem um der ab 1967 wirksam werdenden IDBWeisung an ihre Kreditnehmer, nur noch Lieferanten in anleihensgewährenden Ländern zu berücksichtigen. Bis heute konnte die IDB auf dem schweizerischen Kapitalmarkt über 2 Mia Franken aufnehmen (Léchot, 1984). Die Schweiz ist der zweitgrösste Anleihensmarkt der IDB hinter den USA. Die IDB-Kapitalaufnahmen sind durch die nichteinbezahlten Kapitalquoten der USA und der andern Mitglieder vollumfänglich gedeckt. Seit 1976 ist die Schweiz Mitglied der IDB und verfügt über einen Sitz im Gouverneursrat.

\section{Weitere Beiträge der Schweiz im internationalen Finanzsystem}

Die Verschuldung Lateinamerikas kann bekanntlich zu einem sehr ernsten Problem werden, wenn die Mehrzahl der Gläubiger zu weiteren Krediten nicht mehr bereit wäre. Umgekehrt erfordert aber die Kreditfortsetzung gewisse Aenderungen der Strategien dieser Länder, da andernfalls die Verschuldungsprobleme bis in fünf oder zehn Jahren voraussichtlich noch viel dramatischere Dimensionen annehmen als sie heute aufweisen. Der IWF hat in den letzten Jahren einerseits als Schrittmacher des Vertrauens gewirkt - ein IWF-\$ löste $4 \$$ anderer Finanzquellen aus (Bundesrat, 1984) - und anderseits wirtschaftspolitische Auflagen gemacht. Ohne Zweifel lassen sich diese - besonders was die Haushaltsdefizite der Schuldnerländer angeht - vertreten, aber politisch nicht immer verkraften. Vor allem sind sie wenig dazu angetan, langfristig jene relativen Preise der Produktionsfaktoren und der handelbaren Güter und Dienstleistungen herbeizuführen, die automatisch zu Strategieänderungen führen müssten. Dies würde eine spürbare Veränderung der Wechselkurspolitik einschliesslich der Zollpolitik und anderer Importe und Exporte betreffenden Interventionen der lateinamerikanischen Behörden voraussetzen. Aber es leuchtet ein, dass strukturelle Veränderungen Zeit beanspruchen; bis dieser Prozess der Anpassung über situationskonformere relative Preise durchschlägt, werden noch manche Löcher in den Zahlungsbilanzen gestopft werden müssen. Die schweizerische Nationalbank äusserte stets ihre Bereitschaft, im Krisenfall Liquiditätshilfe zu leisten (Institutional Investor, Feb. 1983). So wirkte auch die Schweiz bei den Ueberbrückungskrediten der "Bank für Internationalen Zahlungsausgleich" (BIZ) an Lateinamerika mit. 
Auf Bundesebene erfolgte 1983 mit einem verfügbaren Kreditpotential von 2,3 Mia Franken der Beitritt zu den erweiterten allgemeinen Kreditvereinbarungen (AKW) des IWF; diese sind als Reserve des IWF für Notfälle gedacht.

Die Schweiz arbeitet auch im Rahmen der im "Pariser Club" geführten Umschuldungsverhandlungen mit. Dort geht es um die Konsolidierung staatlich garantierter kommerzieller Kredite, wie sie im Falle der Schweiz durch die Garantien der ERG auftreten.

\section{Perspektiven}

Da die heutige Schulden-Situation als Folge der Ueberlagerung sehr zahlreicher interner und externer Prozesse eine harte Realität darstellt, die in mehr als 20 Jahren kumulativ entstand, müssen auch die Lösungen realistisch sein. Dazu gehört in erster Linie die Einsicht, dass die Schuldnerländer nicht aus eigener Kraft die Probleme meistern können. Die Finanzminister der wichtigsten lateinamerikanischen Länder formulierten unlängst ihre Vorstellung (NZZ, 25.6.84). :

- Zinsstabilisierung bzw. -plafonierung,

- längerfristige Strukturierung der Kredite,

- längere Kreditperioden ohne Amortisation,

- Festlegung einer Schuldendienstquote im Verhältnis zu den Exporterlösen,

- Neue Kredite,

- Oeffnung von geschützten Märkten in Industrieländern.

Eine für den schweizerischen Bank- und Industriesektor repräsentative Umfrage zur internationalen Verschuldungskrise (Stellungnahme, 1984) stimmt im wesentlichen mit den lateinamerikanischen Forderungen überein. Die Schweizerseite betont dabei auch besonders die Notwendigkeit monetärer und fiskalpolitischer Disziplin in Lateinamerika und erhofft den Abbau vieler Behinderungen gegenüber dem Auslandskapital. Die lateinamerikanischen Partner sollten nebst diesen Eckwerten neue entwicklungspolitische Felder bestellen, welche sich vor allem ausserhalb der heutigen formellen Sektoren befinden und auch die Beziehungen zwischen dem modernen und dem informellen Sektor einschliessen. Mut zu mehr kleineren Projekten, die eine gute Vernetzung aufweisen, und einzeln gesehen nicht so kostspielig sind wie die immer grösser werdenden industriellen und infrastrukturellen Vorhaben, wird nötig sein. Wenn als Kosten der benutzten Produktionsfaktoren Arbeit, Kapital und Energie deren wirkliche Preise eingesetzt würden, ergäben sich ohne Zweifel bald attraktive Rentabilitäten auch in Projekten privater und öffentlicher Art, die bislang - wenn auch im kleineren Massstab - vor allem durch Organe der internationalen Entwicklungszusammenarbeit durchgeführt werden. 
Die Schweiz ist im Verhältnis zu ihrer Grösse und vergleichsweise zu andern Industriestaaten in Lateinamerika wirtschaftlich überaus stark vertreten. Dies macht sie - auch aus verständlichem Eigeninteresse - zu einem soliden, an langfristig günstigen Entwicklungen interessierten Partner Lateinamerikas. Dies ist in schwierigen Zeiten wie heute besonders wichtig. Für die Gestaltung der Beziehungen unseres Landes zu Lateinamerika im öffentlichen und im privaten Sektor werden künftig die Aspekte der Ausgeglichenheit (Breiteneffekte) sowie der angemessenen Schnelligkeit (Verdaubarkeit) der Entwicklungen in Lateinamerika an Bedeutung stark gewinnen.

\section{Literaturverzeichnis}

(ohne Tages- und Wochenzeitungen, die im Text direkt zitiert werden)

Botschaft betreffend den Abschluss von Abkommen über den Schutz und die Förderung von Kapitalinvestitionen vom 17.11.1982.

Bundesrat : Bericht über die Risiken der internationalen Verschuldung vom 12.3.1984.

Enderlin, Hanspeter : Strukturelle Wandlungen in den Wirtschaftsbeziehungen der Schweiz mit Lateinamerika, Diss. Univ. Zürich 1973.

Esser, Klaus \& von Gleich, Albrecht (Hrsg.) : Lateinamerika - Entwicklungsprozess am Wendepunkt?, Hamburg 1984.

Interamerican Development Bank : div. Jahresberichte.

Latin American Bureau : The European Challenge, Europe's New Role in Latin America, London 1982.

Léchot, E.H. : Aperçu des relations commerciales entre la Suisse et l'Amérique latine, 2 Vorträge vom 29.3.1983 und vom 21.3.1984.

Leutwiler, Fritz: Gedanken zur internationalen Verschuldung, Vortrag vom 6.9.1983 in Wien, Verband österreichischer Bankiers und Banken.

OZD : Schweizerische Aussenhandelsstatistik, Jahresbericht der entsprechenden Jahre (Eidg. Oberzolldirektion).

Petersen, Hans J. (Hrsg.) : Die Beziehungen zwischen der Europäischen Gemeinschaft und Lateinamerika, Nomos, Baden-Baden 1983.

SALA (Statistical Abstract of Latin America), Vol. 22, Los Angeles 1981.

Schweizerisch-Lateinamerikanische Handelskammer : Kurier, div. Nr.

Schweizerische Zentrale für Handelsförderung : Auslandsmärkte, Sonderausgabe : 100 Handelspartner, 1984.

Stellungnahme : Internationale Verschuldungskrise - eine schweizerische Stellungnahme, Die Bank, Nr. 3/1984. 